\title{
Anomalous retroreflection from strongly absorbing nanoporous semiconductors
}

\author{
S. Prislopski ${ }^{1}$, E. Naumenko ${ }^{1}$, I. Tiginyanu ${ }^{2,3}$, L. Ghimpu ${ }^{2}$, E. Monaico ${ }^{3}$, L. Sirbu ${ }^{2}$, \\ S. Gaponenko ${ }^{1}$ \\ B. I. Stepanov Institute of Physics, National Academy of Sciences of Belarus, Belarus; \\ ${ }^{2}$ Institute of Electronic Engineering and Nanotechnologies "D. Ghitu”, Academy of Sciences of Moldova, \\ Moldova; \\ ${ }^{3}$ National Centre for Materials Study and Testing, Technical University of Moldova, Moldova
}

Pronounced retroreflection behavior is reported for a fishnet nanoporous strongly absorbing semiconductor material. Retroreflection features a half-cone about 0.35 rad along with diffusive specular reflection for all angles of incidence. Retroreflection is apparent by the naked eye with daylight illumination and exhibits no selectivity with respect to wavelength and polarization of incident light featuring minor depolarization of retroreflected light. The reflectance in the backward direction measures $12 \%$ with respect to a white scattering etalon. The phenomenon can be classified neither as coherent backscattering nor as Anderson localization of light. The primary model includes light scattering from strongly absorptive and refractive superwavelength clusters existing within the porous fishnet structure. A reasonable qualitative explanation is based on the fact that strict retroreflection obeys shorter paths inside absorbing medium, whereas all alternative paths will lead to stronger absorption of light.

NOTES

\section{Comparative electrooptical response from zero-, one, and two-dimensional CdSe nanocrystals}

\author{
A. Prudnikau ${ }^{2}$, M. Artemyev ${ }^{1}$, M. Ermolenko ${ }^{2}$, S. Gaponenko ${ }^{2}$ \\ ${ }^{1}$ Institute for Physico-Chemical Problems of Belarusian State University, Belarus; \\ ${ }^{2}$ B.I Stepanov Institute of Physics, Belarusian Academy of Sciences, Belarus
}

We synthesized by colloidal chemistry way the quantum confined CdSe nanocrystals of different dimensionality: 0D (quantum dots), 1D (nanorods), 2D (platelets). CdSe nanocrystals have been introduced into the polymeric films sandwiched between two transparent conductive electrodes. The room temperature electrooptical effects were studied in form of differential absorption spectra of nanocrystals versus the applied electric field. We observed an order of magnitude larger electrooptical response from CdSe platelets, as compared to quantum dots, while, CdSe nanorods show a little effect. The mechanisms for dimensionalitydependent electrooptical effect in semiconductor nanocrystals, as well as possible practical applications are discussed in the presentation.

NOTES

\section{Main properties of effective dimensional reduction for relativistic Dirac particles}

\author{
A. Silenko, O. Teryaev \\ Research Institute for Nuclear Problems, Belarus; \\ Joint Institute for Nuclear Research, Russia
}

We consider an example of an effective dimensional reduction for a Dirac particle in a curved spacetime and use it for investigation of general properties of Dirac particles in effective two-dimensional spaces. The reduction is just effective because the particle remains in the three-dimensional space while one of its dimensions is compactified. This is assumed to be a model for the generic low-dimensional structures. We perform the Foldy-Wouthuysen transformation of the covariant Dirac equation for three spatial dimensions and determine general properties of momentum and spin dynamics at the above reduction. We use the specific metric admitting such a reduction. We derive the corresponding relativistic Foldy-Wouthuysen Hamiltonian and equations of motion. We formulate general conditions and find general properties of the effective dimensional reduction. We find the quantum mechanical and semiclassical equations of spin motion. For a particle in a curved spacetime, the axis of spin quantization coincides with the axis corresponding to the compactified dimension. However, the direction of this axis can be changed by an additional electromagnetic interaction. For this case, the semiclassical and classical limits are studied. The work is supported by the Belarusian Republican Foundation for Fundamental Research (Grant No. Ф10Д-001). 